\title{
Assessing the impact of complications on the costs of Type II diabetes
}

\author{
R. Williams' ${ }^{1}$ L. Van Gaal' ${ }^{2}$, C. Lucioni ${ }^{3}$ \\ ${ }^{1}$ Nuffield Institute for Health, Division of Public Health, University of Leeds, Leeds, UK \\ 2 Flemish Diabetes Association, Vlaamse Diabetes Vereniging, Belgium \\ ${ }^{3}$ Institute of Health Economics, Institute of Economics Sanitaria, Milan, Italy
}

\section{Abstract}

Aims/hypothesis. 'The Cost of Diabetes in Europe Type II (CODE-2) study' provides the first coordinated attempt to assess the total costs of managing people with Type II (non-insulin-dependent) diabetes mellitus in Europe. Type II diabetes is associated with a number of serious long-term complications, which are a major cause of morbidity, hospitalisation and mortality in diabetic patients.

Methods. Patients were divided into four broad categories defining their complication status in terms of no complications, one or more microvascular complications, one or more macrovascular complications or one or more of each microvascular and macrovascular complication. The prevalence of complications and associated costs were assessed retrospectively for 6 months.

Results. In total, $72 \%$ of patients in the CODE- 2 study had at least one complication, with $19 \%$ having microvascular only, $10 \%$ having macrovascular only and $24 \%$ of the total having both microvascular and macrovascular complications. Of patients with microvascular complications, $28 \%$ had neuropathy, $20 \%$ renal damage, $20 \%$ retinopathy and $6.5 \%$ required treatment for eye complications. Among the patients with macrovascular complications, $18 \%$ had peripheral vascular disease, $17 \%$ angina, $12 \%$ heart failure and $9 \%$ had myocardial infarction. Percutaneous transluminal coronary angioplasty, coronary artery bypass graft or stroke occurred in 3\%, $4 \%$ and $5 \%$ of the patients, respectively. In patients with both microvascular and macrovascular complications, the total cost of management was increased by up to $250 \%$ compared to those without complications.

Conclusion/interpretation. Complications have a substantial impact on the costs of managing Type II diabetes. This study has confirmed that the prevention of diabetic complications will not only benefit patients, but potentially reduce overall healthcare expenditure. [Diabetologia (2002) 45:S13-S17]

Keywords Type II diabetes, CODE-2, complication, costs, management, pharmacoeconomics.
Published online: 12 June 2002

(C) Springer-Verlag 2002

The authors wrote on behalf of the CODE-2 advisory board Corresponding author: Dr. R. Williams, Nuffield Institute for Health, Division of Public Health, 71-75 Clarendon Road, Leeds LS2 9PL, UK, E-mail: d.r.r.williams@leeds.ac.uk Abbreviations: CODE-2, Cost of diabetes in Europe-Type 2; ADA, American Diabetes Association; MI, myocardial infarction; HRQoL, health related quality of life; PVD, peripheral vascular disease; PTCA, percutaneous transluminal coronary angioplasty; CABG, coronary artery bypass graft; TIA, transient iscaemic attack.
Type II (non-insulin-dependent) diabetes mellitus develops as part of a wider metabolic syndrome that includes central obesity, dyslipidaemia (particularly hypertriglyceridaemia and low high density lipoprotein (HDL) cholesterol concentrations), impaired glucose tolerance, coronary artery disease and hyperinsulinaemia, collectively termed 'Syndrome- $X$ ' or 'dysmetabolic syndrome' [1]. The link between this cluster is now well established. Hyperinsulinaemia represents a compensatory response to impaired insulin action [2] and it is generally agreed that underlying insulin resistance precedes the onset of the disease state and also predicts its future development. 
Table 1. The chronic complications of diabetes

\begin{tabular}{|c|c|c|}
\hline System affected & Disease & Potential economic implications \\
\hline Eyes & $\begin{array}{l}\text { Retinopathy, Glaucoma } \\
\text { Cataracts } \\
\text { Blindness }\end{array}$ & $\begin{array}{l}\text { Photocoagulation therapy } \\
\text { Social assistance, loss of productivity } \\
\text { Cataract removal surgery, Drug therapy }\end{array}$ \\
\hline Blood vessels & $\begin{array}{l}\text { Coronary artery disease (CAD) } \\
\text { Cerebral vascular disease (CVD) } \\
\text { Peripheral vascular disease (PVD) } \\
\text { Hypertension }\end{array}$ & $\begin{array}{l}\text { Drug therapy } \\
\text { Emergency hospitalisation } \\
\text { Hospitalisation } \\
\text { Surgical intervention (CABG, PTCA) } \\
\text { Home help/living assistance } \\
\text { Death - loss of productivity }\end{array}$ \\
\hline Kidneys & $\begin{array}{l}\text { Renal insufficiency } \\
\text { Kidney failure }\end{array}$ & $\begin{array}{l}\text { Drug therapy } \\
\text { Hospitalisation - Dialysis } \\
\text { Renal transplantation } \\
\text { Death - loss of productivity }\end{array}$ \\
\hline Skin, Muscle, Bone & $\begin{array}{l}\text { Advanced infections } \\
\text { Cellulitis } \\
\text { Gangrene } \\
\text { Amputation }\end{array}$ & $\begin{array}{l}\text { Drug therapy } \\
\text { Orthotics } \\
\text { Hospitalisation - Surgery } \\
\text { Rehabilitation }\end{array}$ \\
\hline
\end{tabular}

As a consequence of its complex pathophysiology, Type II diabetes is frequently associated with longterm complications (Table 1); both of macrovascular and microvascular origin. Macrovascular disease affecting the heart and large blood vessels, is probably the major cause of both morbidity and mortality in diabetic patients. The American Diabetes Association (ADA) suggest that diabetic patients are 2 to 4 times more likely to suffer heart disease or stroke than nondiabetic patients [3, 4]. A large, growing body of evidence clearly implicates risk factors associated with hyperglycaemia, hyperlipidaemia and insulin resistance in the development of coronary artery disease and mortality in Type II diabetic patients [5]. The 'hyperinsulinaemia cluster' which includes high BMI, triglycerides and insulin with low HDL cholesterol, was shown to be predictive of death [6]. A recent population-based study in Italy assessed the impact of diabetes, showing increased mortality in Type II diabetic patients which could be attributed to raised baseline fasting glucose $(>8.89 \mathrm{mmol} / \mathrm{l})$ [7]. In those patients with hypertension the risk of death from cardiovascular disease was increased by $40 \%$, thus reiterating the negative impact of this condition in diabetic patients.

Complications of microvascular origin include diabetic retinopathy, nephropathy and neuropathy. Diabetic retinopathy is the leading cause of new cases of blindness and visual disability in adults in economically developed societies. Loss of vision due to certain types of glaucoma and cataract could also be more common in people with diabetes than in those without the disease. In the majority of all end-stage renal failure requiring dialysis and renal transplant, diabetic nephropathy has been the underlying condition $[8,9]$. Diabetic neuropathy is less well defined although studies suggest that more than $50 \%$ of people with diabetes are affected to some degree. The largest contribution to morbidity from diabetic neuropathy is due to complications of the feet [10]. Healing-resistant foot ulceration frequently leads to hospitalisation and amputation and its treatment makes it one of the most costly complications of diabetes. It has been shown that the risk of lower extremity amputation is 15 to 40 times higher in diabetic patients $[3,4]$.

The management of diabetes alone renders considerable expenditure, however, macrovascular and microvascular complications are the major cause of healthcare costs $[11,12,13,14]$. Data from a United States study, indicate that renal and cardiovascular complications seem to be the most prevalent and were associated with particularly high costs [14]. In this study abnormal renal function and end-stage renal disease were shown to vastly increase costs of diabetes treatment, up to $771 \%$. Furthermore, an analysis of several individual studies showed that atherosclerosis in Type II diabetes accounted for approximately one third of the total healthcare costs related to the disease [15]. In a study of patients with myocardial infarction (MI), patients with diabetes had a higher per-patient total direct medical charge (inclusive of initial hospitalisation) compared to patients without diabetes [16].

Although data from isolated studies in the United States provide an indication of the scale of the problem, the relative paucity of data generated in Europe has stimulated the initiation of our study. 
Table 2. Inclusion criteria for microvascular and macrovascular complications of Type II diabetes for the CODE 2 study

\begin{tabular}{ll}
\hline Microvascular & Macrovascular \\
\hline Foot ulcer & Angina \\
Amputation & MI \\
Retinopathy & Heart failure \\
Photocoagulation & PTCA \\
Vitrectomy & CABG \\
Blindness (one/both eyes) & TIA \\
Microalbuminuria & Stroke \\
Nephropathy & PVD \\
Dialysis & \\
Renal transplant & \\
Neuropathy & \\
\hline
\end{tabular}

\section{Subjects and methods}

The CODE-2 study provides the first coordinated attempt to assess the total costs of managing patients with Type II diabetes in European countries. This prevalence-based, bottom-up study, involved over 7000 patients in eight European countries (Belgium, France, Germany, Italy, the Netherlands, Spain, Sweden and the UK). The methodology for the CODE-2 study is presented elsewhere in this supplement [17]. The primary objective was to measure and compare the costs of patients with Type II diabetes in each country studied. Secondary objectives included assessing the economic impact of the longterm complications of diabetes and the impact on patients' health-related quality of life (HRQoL) [18].

The prevalence of Type II diabetes complications was assessed by completing a medical practitioners survey designed to encompass all aspects of the long-term consequences and treatments of Type II diabetes. The impact of HRQoL is reported in this supplement [18]. Retrospective collection of this data over a minimum period of 6 months included an assessment of the overall prevalence of complications and incidence of events related to complications. Data on the management and treatment of these complications was also collected, and allowed estimates of total healthcare costs of patients by complication status.

Classification of complications. For analysis, people were classified into four broad categories of complication status: no complications, one or more microvascular complication only, one or more macrovascular complication only, one or more of each of microvascular and macrovascular complications.

The individual conditions and interventions defining microvascular and macrovascular complication status are detailed in Table 2. It should be noted that in previous studies, foot ulcers and amputation have been considered to be of both microvascular and macrovascular origin [10]. However, for the analysis, these conditions were assigned to the microvascular group.

\section{Results}

Prevalence of complications. Of the total CODE-2 study population, $72 \%$ of subjects were reported to have at least one complication, $19 \%$ had only microvascular complications, $9.6 \%$ had only macrovascular complications and $24 \%$ had both microvascular and macrovascular complications. The prevalence of microvascular and macrovascular complications in the

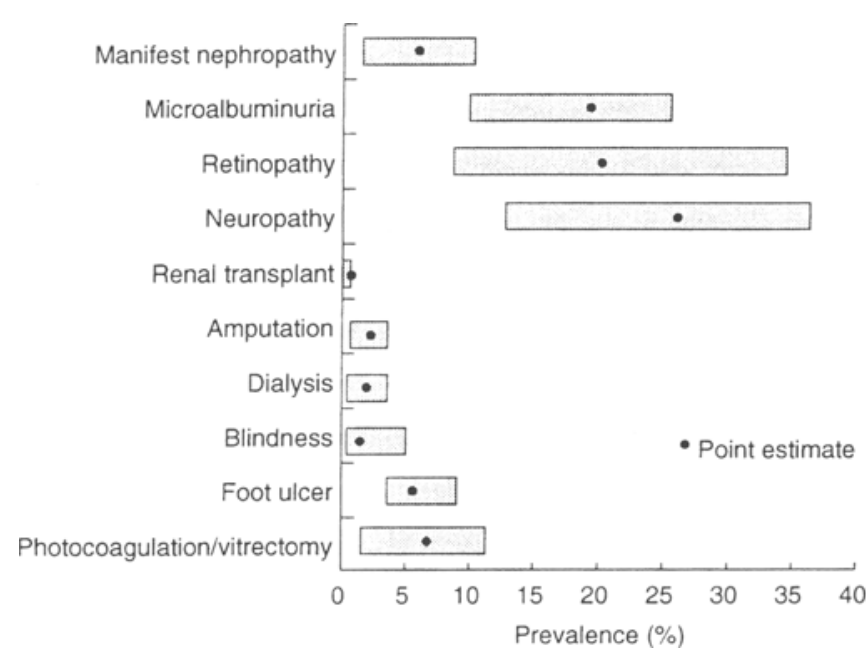

Fig. 1. Prevalence of microvascular complications in patients with Type II diabetes

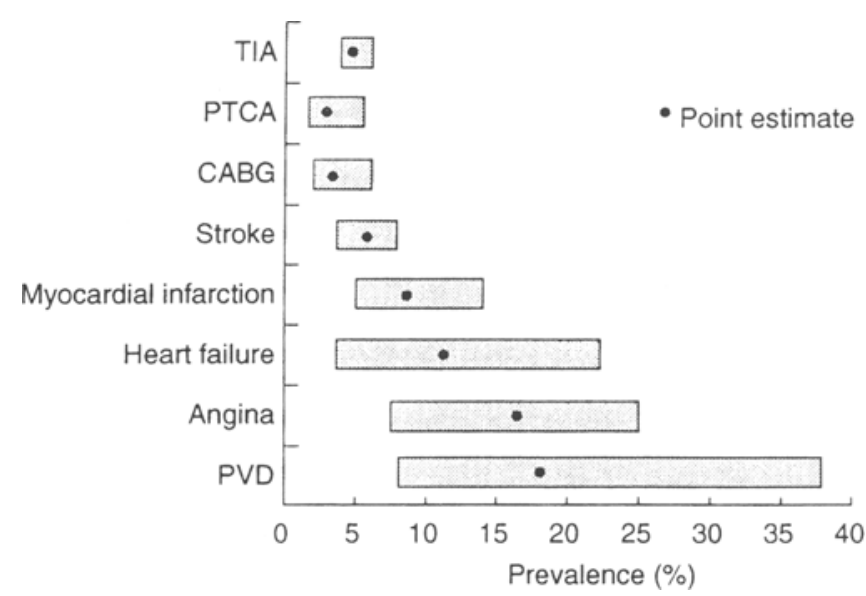

Fig. 2. Prevalence of macrovascular complications in patients with Type II diabetes

CODE-2 population, weighted for the size of each national contribution, are shown in Figs. 1 and 2, respectively. A total of $28 \%$ of the patients with microvascular complications had neuropathy, $20 \%$ showed early signs of renal damage (microalbuminuria), $20 \%$ retinopathy and $6.5 \%$ required treatment for sightthreatening eye complications in the previous 6 months. Furthermore, $2 \%$ of the patients were blind or had lower extremity amputation, and $1 \%$ had end-stage renal failure requiring dialysis.

Of the $33 \%$ of patients with macrovascular complications, $18 \%$ had peripheral vascular disease (PVD), $17 \%$ had angina, $12 \%$ had a history of heart failure and $9 \%$ had history of MI. Vascular reconstruction surgery, such as percutaneous transluminal coronary angioplasty (PTCA) and coronary artery bypass graft (CABG), or stroke occurred in $3 \%, 4 \%$ and $5 \%$ of the patients, respectively.

Incidence of complications. Foot ulcers and sightthreatening retinopathy accounted for the highest annual incidence $(2 \%)$ of microvascular complica- 


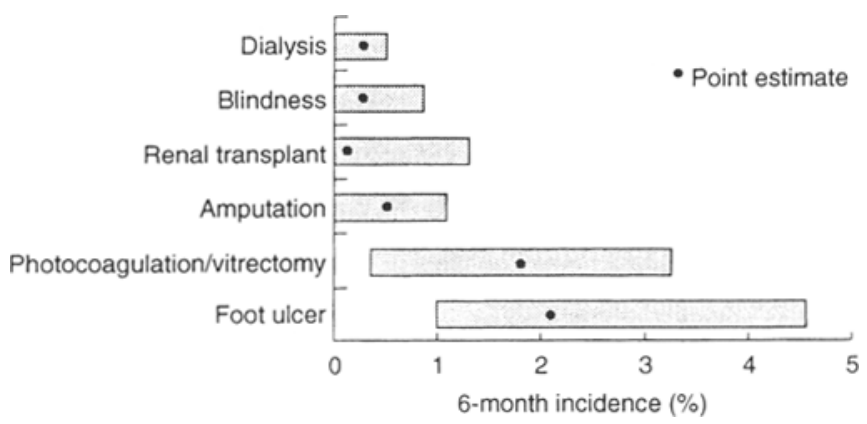

Fig. 3. 6-month incidence of microvascular complications

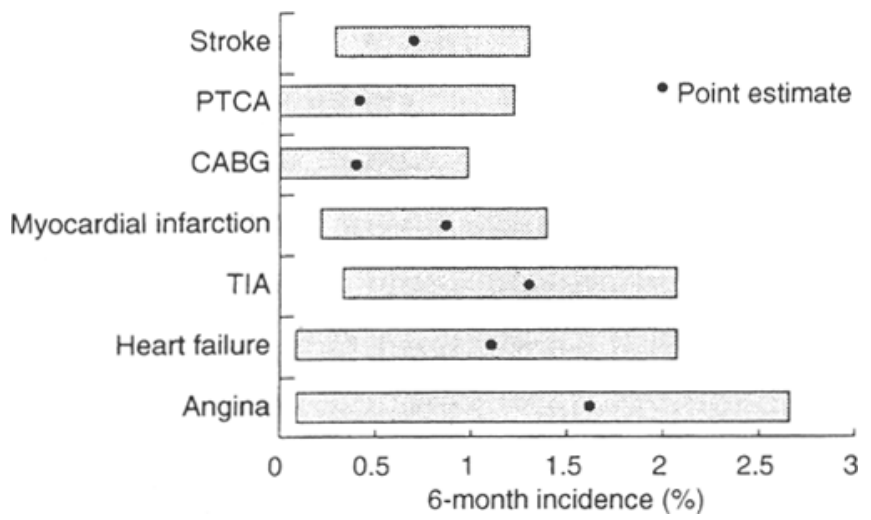

Fig. 4. 6-month incidence of macrovascular complications

tions, followed by amputation $(0.5 \%)$, end-stage renal failure with dialysis $(0.2 \%)$, and blindness $(0.2 \%)$ (Fig. 3). For the macrovascular complication group, angina was observed in $1.6 \%$ of the patients, and heart failure and transient ischaemic attacks (TIA) in 1.1 and $1.3 \%$ of patients, respectively (Fig. 4).

Effect of complications on costs. As expected, analysis of the CODE-2 data confirmed that complications can have an impact on the overall direct medical costs of patients with Type II diabetes. A patient with no complications was found to cost, on average, EUR 1505 per year in direct medical costs. The presence of microvascular complications led to a $70 \%$ increase in costs (EUR 2563) compared to patients with no complications (Fig. 5). Costs for patients with macrovascular complications (EUR 3148) were twice as high as patients with no complications. Those patients with both microvascular and macrovascular complications (EUR 5226), increased costs by 3.5-fold over those without complications. Managing patients with both microvascular and macrovascular complications together represented a marked increase in expenditure, considerably greater than the sum of the cost for each complication category.

A very large proportion of the increase in direct medical cost was attributable to an increase in hospitalisation expenditure (Fig. 6). For patients with only microvascular or macrovascular complications, the incremental increase in hospitalisation cost was $100 \%$ and $200 \%$, respectively, compared with costs for pa-

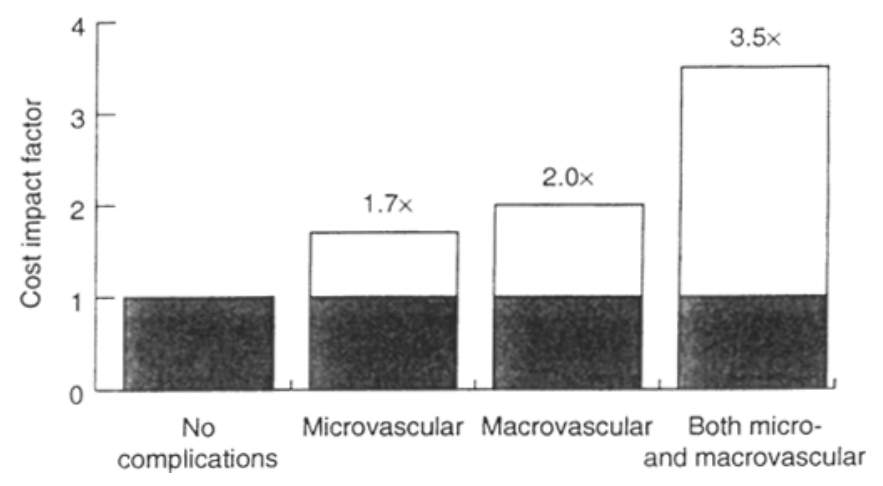

Fig. 5. Effect of complications on the average cost per patient

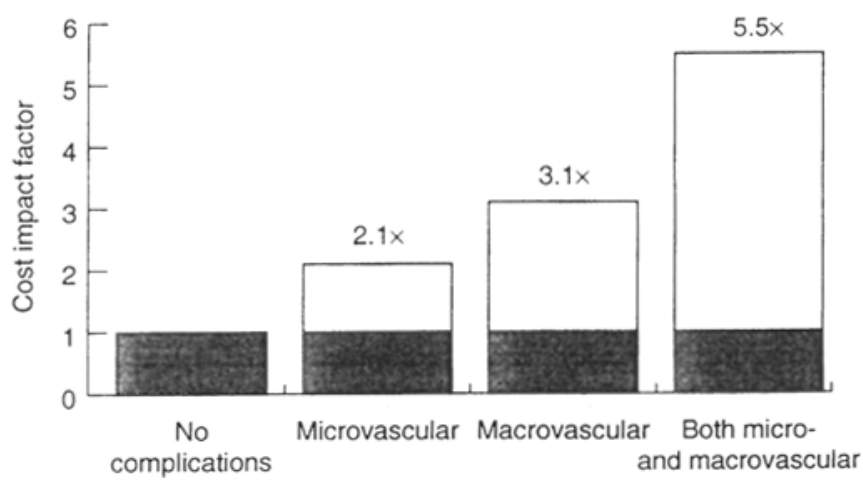

Fig. 6. Effect of complications on hospitalisation costs

tients without complications. However, inpatients with both classes of complications, hospitalisation costs were increased by a total of $450 \%$.

\section{Discussion}

From the CODE-2 study results, it is clear that the presence of complications has a substantial impact on the costs for managing patients with Type II diabetes. For example, in individuals with both microvascular and macrovascular complications, the total cost of management was increased by up to 3.5 times compared to those with no evidence of complications.

Overall, the presence of microvascular complications resulted in an increase of $70 \%$ compared with people with no evidence of complications. The microvascular complications of Type II diabetes include diabetic retinopathy, neuropathy and nephropathy. Retinopathy is associated with considerable costs related to laser coagulation therapy, vitrectomy in severe cases, and eventually costs for social support when useful vision has deteriorated completely [19]. In the CODE-2 study, the prevalence of retinopathy was $20 \%$, while the reported incidence of both photocoagulation and vitrectomy was $2 \%$. This indicated that a large potential for cost savings exists in preventive measures and early retinal screening programmes.

The impact of diabetic neuropathy on the total costs is difficult to assess, although neuropathic conditions are associated with considerable morbidity [20]. Neu- 
ropathy accounted for the highest proportion ( $>25 \%)$ of microvascular complications in the CODE-2 population. Approximately 1 in 200 patients underwent an amputation during the 6-month period of retrospective data collection.

Diabetic nephropathy has been reported to be of relatively low prevalence in Western Type II diabetic populations, probably due to the late onset of the disease in these countries [21]. Nevertheless, the management of end-stage nephropathy consumes resources considerably, requiring dialysis and renal transplantation. As a consequence the impact of nephropathy on the total costs for managing Type II diabetes is likely to be high. Approximately $7 \%$ of the CODE-2 patient population presented with nephropathy, while $20 \%$ were found to have microalbuminuria.

The association between Type II diabetes and the risk of morbidity and mortality due to macrovascular conditions such as angina, MI and stroke is well established $[22,23]$. In the CODE-2 patient population, the overall prevalence of macrovascular complications was found to be up to 20\% (angina and PVD) and 10\% of the patients presented a history of MI or heart failure. Furthermore, the incidence data obtained in the CODE-2 trial showed that a large number of macrovascular complications occurred during the 6-month study period. In total, the presence of macrovascular complications resulted in a cost increase of approximately $100 \%$ compared to patients with no evidence of complications.

In conclusion, the prevention of Type II diabetes related complications will provide benefit to the patients as well as potentially reduce the overall healthcare expenditures for countries and payers. The implementation of the recently published Guidelines for Diabetes Care [24] recommends optimal management of blood glucose, blood pressure and lipid concentrations, and the early detection and management of existing complications to reduce further disease progression.

From the results of this paper on the costs related to complications in conjunction with a paper in this supplement [18], (showing the reduction in HRQoL for patients with these complications), it can be concluded that treatment regimens that reduce these diabetes-related complications can have an impact on the costs of patients with Type II diabetes and also a reduction in patients' well being. Treatment regimens that address the insulin resistance syndrome and also avoid the requirement for initiation of insulin-based regimens can expect to provide a benefit for the patient and potential cost savings for the treatment of Type II diabetes.

Acknowledgements. This study has been financially supported by SmithKline Beecham.

\section{References}

1. Reaven GM (1988) Banting lecture. Role of insulin resistance in human disease. Diabetes 37:1595-1607
2. Reaven GM, Hollenbeck CB, Chen Y-Di (1989) Relationship between glucose tolerance, insulin secretion and insulin action in non-obese individuals with varying degrees of glucose tolerance. Diabetologia 32:52-55

3. Cowie CC, Eberhardt MS (eds) (1996) Diabetes 1996: Vital Statistics. American Diabetes Association, Alexandria

4. American Diabetes Association (1997) Diabetes Facts and Figures. American Diabetes Association, Alexandria

5. Hanefeld M, Schmechel H, Schwanebeck U, Lindner J, The DIS Group (1997) Predictors of coronary heart disease and death in NIDDM: The diabetes Intervention Study Experience. Diabetologia 40:S123-S124

6. Lehto S, Ronnemaa T, Pyorala K, Laasko M (2000): Cardiovascular risk factors clustering with endogenous hyperinsulinaemia predict death from coronary heart disease in patients with Type II diabetes. Diabetologia 43:148155

7. Bruno G, Merletti F, Boffetta P et al. (1999) Impact of glycaemic control, hypertension and insulin treatment on general and cause-specific mortality: an Italian populationbased cohort of Type II (non-insulin-dependent) diabetes mellitus. Diabetologia 42:297-301

8. Cunha-Vaz J (1998) Lowering the risk of visual impairment and blindness. Diabet Med 15:47-50

9. Ritz E (1999) Nephropathy in Type 2 diabetes. J Intern Med 245:111-126

10. Ward JD (1995b) The cost of diabetic foot problems. Pharmacoeconomics 8:55-57

11. Huse DM, Oster G, Killen AR, Lacey MJ, Colditz GA (1989) The economic costs of non-insulin-dependent diabetes mellitus. JAMA 262:2708-2713

12. Susman JL, Helseth LD (1997) Reducing the complications of type II diabetes: a patient-centered approach. Am Fam Physician 56:471-480

13. O’Brien JA, Shomphe LA, Kavanagh PL, Raggio G, Caro JJ (1998) Direct medical costs of complications resulting from Type 2 diabetes in the U.S. Diabetes Care 21:1122-1128

14. Brown JB, Pedula KL, Bakst AW (1999) The progressive cost of complications in Type 2 diabetes mellitus. Arch Intern Med 159:1873-1880

15. MacLeod KM, Tooke JE (1995) Direct and indirect costs of cardiovascular and cerebrovascular complications of Type II diabetes. Pharmacoeconomics 8:46-51

16. Smith TL, Melfi CA, Kesterson JA, Sandmann BJ, Kotsanos JG (1999) Direct medical charges associated with myocardial infarction in patients with and without diabetes. Med Care 37:AS4-AS11

17. Jönsson B (2000) Revealing the cost of Type II diabetes in Europe. Diabetologia Supplement (this issue)

18. Koopmanschap M (2000) Coping with Type II diabetes: the patient perspective. Diabetologia Supplement (this issue)

19. Javitt JC (1995) Cost savings associated with detection and treatment of diabetic eye disease. Pharmacoeconomics 8:3339

20. Ward JD (1995a) The cost of diabetic neuropathy. Pharmacoeconomics 8:52-54

21. Borch-Johnsen K (1995) The costs of nephropathy in Type II diabetes. Pharmacoeconomics 8:40-45

22. Fuller JH, Elford J, Goldblatt P, Adelstein AM (1983) Diabetes mortality: new light on an underestimated public health problem. Diabetologia 24:336-341

23. Head J, Fuller JH (1990) International variations in mortality among diabetic patients: the WHO Multinational Study of Vascular Disease in Diabetics. Diabetologia 33:477-481

24. European Study Group (1999) A desktop guide to Type 2 diabetes mellitus. European Diabetes Policy Group 1999. Diabet Med 16:716-730 